\title{
Drogas emergentes (II): el pharming
}

\section{Emergent drugs (II): the Pharming phenomenon}

\author{
G. Burillo-Putze ${ }^{1}$, A. Aldea-Perona ${ }^{2}$, C. Rodríguez-Jiménez ${ }^{4}$, M.M. García-Sáiz ${ }^{1,2}$, \\ B. Climent ${ }^{3}$, A. Dueñas ${ }^{4}$, P. Munné ${ }^{5}$, S. Nogué ${ }^{5}$, R.S. Hoffman ${ }^{6}$
}

\section{RESUMEN}

El uso por la población joven de fármacos con y sin receta médica con fines recreativos, ha tenido escasa atención por los médicos. En USA, uno de cada cinco adolescentes han usado fármacos con finalidad recreativa, y en el servicio de Urgencias, las consultas por abuso de fármacos han superado a las de drogas ilegales. Aunque es España existen pocos datos, este consumo se sitúa, según las encuestas, entre el 3,1 y el $8,6 \%$.

Los fármacos más utilizados son el dextrometorfán y el metilfenidato. El primero, de venta sin receta, presenta una sintomatología variable, dosis y acción metabólica dependiente, el cual varía desde la euforia a las alucinaciones. El metilfenidato se utiliza como estimulante sustituto de la cocaína, tanto por vía oral como nasal e intravenosa, siendo uno de los fármacos con más desvío hacia el mercado ilícito a nivel mundial.

Otras sustancias como el modafinilo y el propofol presentan un uso no médico en principio de escasa incidencia, pero con un potencial de abuso a tener en cuenta, sobre todo en el ámbito sanitario.

Finalmente, opiáceos como el fentanilo, la oxicodona y la buprenorfina, de reciente generalización en el arsenal terapéutico de muchas especialidades médicas y con nuevas presentaciones farmacéuticas, están produciendo fenómenos de abuso, dependencia y comercio ilícito. Las demandas de tratamiento de desintoxicación, la mezcla con sustancias ilegales y los casos de muerte, alertan sobre el abuso de estos fármacos.

Palabras clave. Pharming. Metilfenidato. Dextrometorfán. Propofol. Modafinilo.

\begin{abstract}
The use of medicines, with or without medical prescription, for recreational ends by the young population has received little attention from doctors. In the USA, one in five adolescents has used medicines for recreational purposes and consultations in Emergency Departments for medicine abuse have exceeded those for illegal drugs. Although few data are available in Spain, such consumption is situated between 3.1 and $8.6 \%$ according to surveys.

The medicines most used are dextromethorphan and methylphenidate. The former, on sale without prescription, presents a varied symptomatology, dosage and dependent metabolic action, ranging from euphoria to hallucinations. Methylphenidate, taken orally, nasally or intravenously, is used as a stimulant in substitution for cocaine and is one of the medicines most diverted onto the illicit market at the world level.

In principle, other substances like modafinil and propofol present a limited incidence of non-medical use, but they have a probable abuse potential that should be borne in mind, above all in the health context.

Finally, opiates like fentanyl, oxycodone and buprenorphine, with new pharmaceutical presentations, have recently become generalized in the therapeutic arsenal of many medical specialities; they are giving rise to phenomena of abuse, dependence and diversion towards the illicit market. Demands for detoxification treatment, their mixture with illegal substances, and cases of death should alert us to the abuse of these medicines.
\end{abstract}

Key words. Methylphenidate. Dextromethorphan. Propofol. Modafinil.
1. Universidad Europea de Canarias, Facultad de Ciencias de la Salud. Servicio de Urgencias. Hospital Universitario de Canarias. Tenerife, España.

2. Servicio de Farmacología Clínica, Hospital Universitario de Canarias, Tenerife. España.

3. Unidad de Toxicología Clínica, Servicio de Medicina Interna, Hospital General Universitario, Valencia, España.

4. Unidad Regional de Toxicología Clínica, Hosital Universitario Río Hortega, Valladolid. España.

5. Sección de Toxicología Clínica, Servicio de Urgencias, Hospital Clìnic, Barcelona, España.

6. New York City Poison Control Center, Bellevue Hospital Center, New York. USA.

\section{Correspondencia:}

Guillermo Burillo Putze

Servicio de Urgencias

Hospital Universitario de Canarias

Ofra s/n La Cuesta. E-38320

La Laguna. Tenerife. España

e-mail: gburillo@telefonica.net

Recepción: 20 de agosto de 2012

Aceptación provisional: 13 de septiembre de 2012

Aceptación definitiva: 30 de octubre de 2012 


\section{EL ALCANCE DEL PHARMING}

En toxicología clínica se aplica el término de pharming (en castellano farming) al consumo de medicamentos con finalidad recreativa, generalmente prescritos para un enfermo diferente al usuario, utilizando una dosis distinta a la indicada como terapéutica, y buscando en general alguno de sus efectos adversos ${ }^{1}$. Este uso como droga de algunos fármacos que en ocasiones requieren receta, pero que también pueden ser de libre dispensación, ha tenido hasta el presente escasa atención médica, si bien se van conociendo datos epidemiológicos sobre este fenómeno. Además, las muertes de artistas como Hetah Ledger en 2008 o Michael Jackson en 2009, relacionadas con el abuso de medicamentos con receta (hipnosedantes por vía oral y propofol intravenoso respectivamente), han supuesto una llamada de atención sobre un problema sanitario probablemente infradiagnosticado ${ }^{2,3}$.

Según el informe de la Junta Internacional de Fiscalización de Estupefacientes correspondiente a 2009, en los últimos 10 años, las muertes por consumo excesivo de medicamentos han aumentado de forma considerable, superando en algunos países a las producidas por el uso de drogas ilegales. A modo de ejemplo, entre 2005 y 2009, el consumo mundial de metilfenidato -medicamento autorizado en España para el trastorno por déficit de atención con hiperactividad (TDAH)- aumentó un 30\%, con casi 40 toneladas de consumo, fundamentalmente en USA ${ }^{4}$.

Una serie de factores influyen en la creciente popularidad del pharming: su fácil accesibilidad tanto desde el botiquín del hogar, como mediante su adquisición directa sin receta en farmacias o a través de internet $^{5}$, el hecho de ser sustancias legales y la percepción de que al ser medicamentos son productos seguros y mucho menos peligrosos que las drogas de la calle, así como la falta de percepción de su uso como drogas por parte de los padres u otros familiares o conocidos. Además de la peligrosidad inherente al consumo abusivo propiamente dicho, el riesgo se incrementa por el hecho de utilizarse varios fármacos de forma con- junta, en muchas ocasiones mezclados con alcohol u otras drogas ${ }^{6}$. En este sentido, se ha observado entre jóvenes estadounidenses el uso del denominado trail mix, consistente en reuniones en las que cada asistente recolecta fármacos del botiquín de su casa, mezclándolos luego en un recipiente, para consumirlos de forma aleatoria ${ }^{2}$.

Otro aspecto a tener en cuenta con el pharming es la posibilidad de que sirva como puerta de entrada al consumo de otras drogas ilegales, como ocurre con el cheese, mezcla de heroína con antigripales (fundamentalmente difenhidramina), que causa euforia y alucinaciones tras inhalarlo, y que es conocida como la "heroína de inicio"7.

En el ámbito sanitario, fue la Dra. Levine, del Bellevue Hospital de Nueva York, la que en 2007 alertó sobre el peligro de esta práctica, tras detectar el fenómeno entre los jóvenes estadounidenses, sobre todo por consumo de productos de venta sin receta (antitusígenos como el dextrometorfán y antigripales con fenilefrina) ${ }^{1}$. Según una encuesta realizada en USA sobre una población de 7.300 niños y adolescentes con edades entre 7 y 12 años, uno de cada cinco adolescentes (19\%) admitió haber usado medicamentos con finalidad recreativa, y el $10 \%$ preparados antigripales, con porcentajes superiores en cualquier caso al de las drogas ilegales. Además, dos de cada cinco encontraban estos productos más seguros que las drogas, y un $30 \%$ consideraba que no eran adictivos ${ }^{1}$.

Según datos de la Red norteamericana de Alerta sobre Abuso de Drogas-Drug Abuse Warning Network-(DAWN), las consultas a Urgencias Hospitalarias por uso incorrecto o abuso de fármacos se incrementaron entre 2004 y 2009 un 98,4\%, llegando a 1.244 .679 casos en el último año. Supusieron el $27,1 \%$ de los casos de uso de drogas y fármacos, superando a las drogas ilegales $(21,2 \%)$ y casi al mismo nivel que el alcohol (29,9\%). En total, el 19,1\% de consultas por uso de fármacos fueron realizadas por menores de 20 años $^{8}$. Los grupos farmacológicos que más se asociaron a las consultas de urgencias fueron los fármacos para el insomnio y la ansiedad (34\%), fundamen- 
talmente benzodiacepinas (86\%), y los analgésicos narcóticos (31\%), mayoritariamente oxicodona (44\%) e hidrocodona (26\%), coincidiendo éstos con algunos de los fármacos que presentaron un mayor incremento de su prevalencia respecto a 2004 (Tabla 1).

Tabla 1. Fármacos con mayor aumento de incidencia de consultas en urgencias entre 2004 y 2009 , según la $D A W N^{8}$.

\begin{tabular}{|l|c|c|}
\hline \multicolumn{1}{|c|}{$\begin{array}{c}\text { Grupo } \\
\text { terapéutico }\end{array}$} & Fármaco & $\begin{array}{c}\text { \% } \\
\text { incremento }\end{array}$ \\
\hline \multirow{2}{*}{$\begin{array}{l}\text { Fármacos } \\
\text { analgésicos }\end{array}$} & Oxicodona & 242,2 \\
\cline { 2 - 3 } & Morfina & 133,3 \\
\cline { 2 - 3 } & Hidrocodona & 124,5 \\
\cline { 2 - 3 } & Fentanilo & 117,5 \\
\hline \multirow{2}{*}{$\begin{array}{l}\text { Fármacos } \\
\text { para el } \\
\text { insomnio y la } \\
\text { ansiedad }\end{array}$} & Zolpiden & 154,9 \\
\cline { 2 - 3 } & Alprazolam & 148,3 \\
\cline { 2 - 3 } & Clonazepam & 114,9 \\
\cline { 2 - 3 } & Lorazepam & 104,3 \\
\hline
\end{tabular}

En España existen pocos datos sobre el fenómeno del pharming ${ }^{9,10}$. En la Encuesta Domiciliaria sobre Alcohol y Drogas en España (EDADES) no comenzó a investigarse el consumo de tranquilizantes y somníferos con o sin receta médica hasta el año 2005. No obstante, entre 2005 y 2007-08 se observó un aumento importante de la prevalencia de consumo durante los últimos 12 meses, pasando de 5,1\% en 2005 a 8,6\% en $2007-08^{11}$.

En 2007-08 en España un 15,4\% de la población de 15-64 años había consumido hipnosedantes con o sin receta médica alguna vez en la vida (13,0\% tranquilizantes, y $4,6 \%$ somníferos), un $8,6 \%$ en los últimos 12 meses $(6,9 \%$ tranquilizantes y $3,8 \%$ somníferos) y un $5,9 \%$ en los últimos 30 días (4,7\% tranquilizantes y $2,5 \%$ somníferos). Además, un 3,1\% los había consumido diariamente en los últimos 30 días (2,6\% tranquilizantes y $1,1 \%$ somníferos $)^{12}$.

Según el Observatorio de Medicamentos de Abuso del Colegio de Farmacéuticos de Barcelona, sistema basado en la notificación voluntaria por estos profesionales de las sospechas de abuso de medicamentos registrados en las oficinas de farmacia y servicios de farmacia de atención primaria y hospitalarios, de un total de 255 notificaciones, para la obtención del medicamento, un $23,13 \%$ lo hicieron mediante demandas sin receta, un $19 \%$ con recetas falsas o manipuladas, y el $48,42 \%$ con una demanda injustificada o continua de fármacos. En un 89,56 \% de casos, el farmacéutico no dispensó el medicamento ${ }^{13}$. Los fármacos involucrados se presentan en la tabla 2 .

Tabla 2. Fármacos objeto de notificación. Observatorio de medicamentos de abuso. Colegio de Farmacéuticos de Barcelona ${ }^{13}$.

\begin{tabular}{|l|l|c|}
\hline \multicolumn{1}{|c|}{ Tipo de sospecha de abuso } & \multicolumn{1}{|c|}{ Fármaco } & $\%$ \\
\hline \multirow{4}{*}{ Uso recreativo } & Codeína & 20,63 \\
\cline { 2 - 3 } & Metilfenidato & 11,11 \\
\cline { 2 - 3 } & Alprazolam & 10,32 \\
\cline { 2 - 3 } & Dextrometorfán & 3,17 \\
\cline { 2 - 3 } & Cloruro de etilo & 2,38 \\
\cline { 2 - 3 } & Ketamina & 1,59 \\
\cline { 2 - 3 } & Otros & 50,02 \\
\hline \multirow{4}{*}{ Uso no indicado } & Misoprostol & 4,76 \\
\hline \multirow{2}{*}{ Fármacos para fabricar otras drogas } & Efedrina/pseudoefedrina & 2,38 \\
\cline { 2 - 3 } & Piracetam & 0,79 \\
\hline
\end{tabular}


Existen otros puntos que pueden ejercer atalaya para la detección del potencial abuso de estos medicamentos con finalidad lúdica, y los cuales han resultado muy útiles en la detección de problemas de salud pública emergentes que pueden pasar desapercibidos a otros niveles asistenciales, como así lo han demostrado en diversas ocasiones los servicios de urgencias hospitalarios $^{14-18}$ y los sistemas de emergencias médicas ${ }^{19,20}$. Y ello es así porque muchos de los efectos secundarios que puede ocasionar el pharming puede conllevar la necesidad de consulta inmediata a estos importantes eslabones de la continuidad médica asistencial ${ }^{21,22}$. No en balde, los SUH y SEM han sido los primeros en detectar casos de abuso de $\mathrm{GHB}^{23}$, de brotes importantes de toxinfección alimentaria ${ }^{24}$ o de gripe $\mathrm{A}^{25}$. No obstante, una búsqueda exhaustiva no ha permitido encontrar referencias al respecto en nuestro país ${ }^{10,26}$ como si en cambio sucede en otros entornos $^{27-29}$ Por ello, y a pesar de su alta carga asistencial y su frecuente saturación ${ }^{30,31}$, los profesionales de estos servicios deben ser especialmente conocedores de esta problemática, un hecho más que justifica la presente revisión.

\section{CLASIFICACIÓN}

Se pueden englobar los fármacos utilizados fundamentalmente por adolescentes como drogas en tres grandes grupos ${ }^{1}$ : los estimulantes (dextrometorfán, metilfenidato) (Fig. 1), los analgésicos opiáceos (fentanilo y oxicodona, entre otros) (Fig. 2), y los hipnosedantes (benzodiacepinas, y las $z$-drugs zolpidem y zopiclona ${ }^{32}$ ). En esta revisión se describen las aspectos toxicológicos y clínicos de los nuevos fármacos de cada uno de estos grupos, dejando de lado aquellas sustancias como los mórficos de uso habitual y las benzodiacepinas, así como otros fármacos utilizados en el ámbito hospitalario o en clínica veterinaria que tuvieron su boom como drogas de diseño al inicio de este siglo (gamma-hidroxi-butirato-GHB-, ketamina, óxido nitroso) y que ya han sido específicamente estudiados en otros trabajos ${ }^{33-35}$.

\section{Metilfenidato}

Aunque el uso clínico de los estimulantes se inició en el siglo XIX tras descubrir los efectos psicoactivos de la cocaína y la anfetamina, no fue hasta el año 1944 cuando el metilfenidato (MTF) fue sintetizado e inicialmente indicado para la fatiga crónica, letargia, estados depresivos, conducta senil perturbada, psicosis asociada a depresión y narcolepsia ${ }^{36}$. En la actualidad, su uso ha quedado restringido al TDAH en niños mayores de 6 años y adolescentes, cuando otras medidas son insuficientes ${ }^{37}$. En España, la única indicación autorizada del MTF es el TDAH y está comercializado como formulaciones de liberación inmediata (Rubifen ${ }^{\circledR}$ y Medicebran ${ }^{\circledR}$ ) o prolongada (Concerta ${ }^{\circledR}$ y Medikinet cápsulas ${ }^{\circledR}$ ).

El MTF es una feniletilamina, y que actúa como estimulante del sistema nervioso central. A pesar de su similitud con las anfetaminas, difiere en sus mecanismos de acción neuroquímicos, en sus efectos sobre la excreción urinaria de catecolaminas y en la respuesta clínica de algunos pacientes. Su mecanismo de acción específico en el TDAH no se conoce con precisión, aunque podría consistir en el bloqueo de la recaptación de noradrenalina y dopamina en la neurona presináptica, incrementando por tanto la concentración de ambos neurotransmisores en el espacio extraneuronal ${ }^{36,37}$.

En los últimos años ha habido un aumento en el diagnóstico de TADH y en la prescripción de psicoestimulantes, multiplicándose por cinco la prescripción en Estados Unidos respecto a los años noven$\mathrm{ta}^{38}$. El tratamiento con MTF requiere una vigilancia estrecha no sólo por los posibles efectos adversos cardiovasculares y la exacerbación de trastornos psiquiátricos ${ }^{36,37}$, sino también por el riesgo de abuso, mal uso o tráfico de MTF.

Los adolescentes abusan de este medicamento por diferentes motivos: como ayuda para concentrarse en los estudios ${ }^{39}$, para incrementar su estado de alerta y de euforia y, como ya se ha comentado, con la falsa creencia de que su abuso es más seguro al ser adquirido por la prescripción de un médico y ajenos al consumo de drogas de 

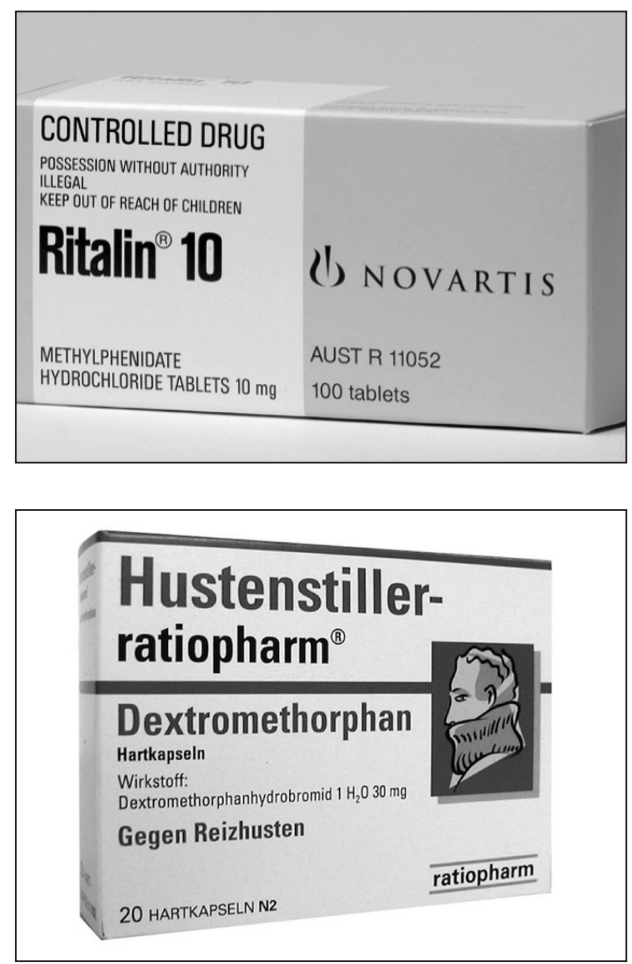

Figura 1. Farmacos usados por su efecto estimulante.

la calle ${ }^{40}$. En una encuesta autocumplimentada por estudiantes canadienses realizada en 1998, de los que contestaron que habían hecho uso de estimulantes con receta en el año anterior, el 14,7\% había dado medicación a sus amigos, y el 7,3\% había vendido algunos de sus estimulantes prescritos, haciéndolo alrededor del $80 \%$ mediante venta a distancia ${ }^{41}$. En USA, el 9\% de los adolescentes entre 12 y 17 años que contestaron a la Encuesta Nacional Sobre el Uso de Drogas -National Survey on Drug Use and Health(NSDUH), desviaron los medicamentos recetados hacia un propósito no médico, de los que el $2 \%$ eran psicoestimulantes. En 2004, la tasa anual de visitas de pacientes de 12 a 17 años al servicio de Urgencias fue de 3,3 por 100.000 habitantes (1,7 para uso no médico) y de 4 por 100.00 habitantes para la anfetamina-dextroanfetamina (2,8 para uso no médico $)^{42}$.

La administración intranasal o intravenosa produce un efecto rápido y similar a
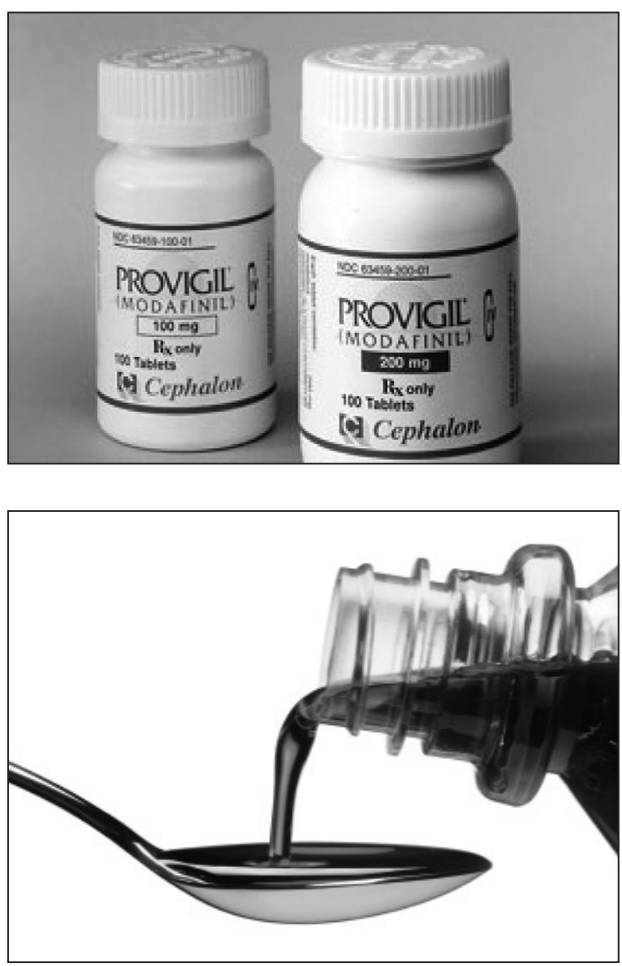

los efectos de la cocaína, debido a una rápida liberación de dopamina presináptica que da lugar a efectos subjetivos de "subidón" e intensa euforia gratificante ${ }^{43}$. En 1967 se publicó el primer caso de psicosis asociado al abuso intravenoso de $\mathrm{MTF}^{44}$ y en 1991, el primer caso de abuso intranasal ${ }^{45}$.

El abuso oral de MTF se ha descrito en la literatura con menos frecuencia. El primer caso se sospechó en los años 60, por el consumo de 125 comprimidos diarios por un paciente. Los posteriores casos de abuso publicados, estaban relacionados con la aparición de alucinaciones, paranoia, euforia y trastorno delirante ${ }^{43}$.

La sobredosis aguda debida fundamentalmente a la hiperestimulación de los sistemas nervioso central y simpaticomimético, puede provocar vómitos, agitación, temblores, hiperreflexia, calambres musculares, convulsiones, coma, euforia, confusión, alucinaciones, delirio, sudoración, rubor, cefalea, hiperpirexia, taquicardia, 

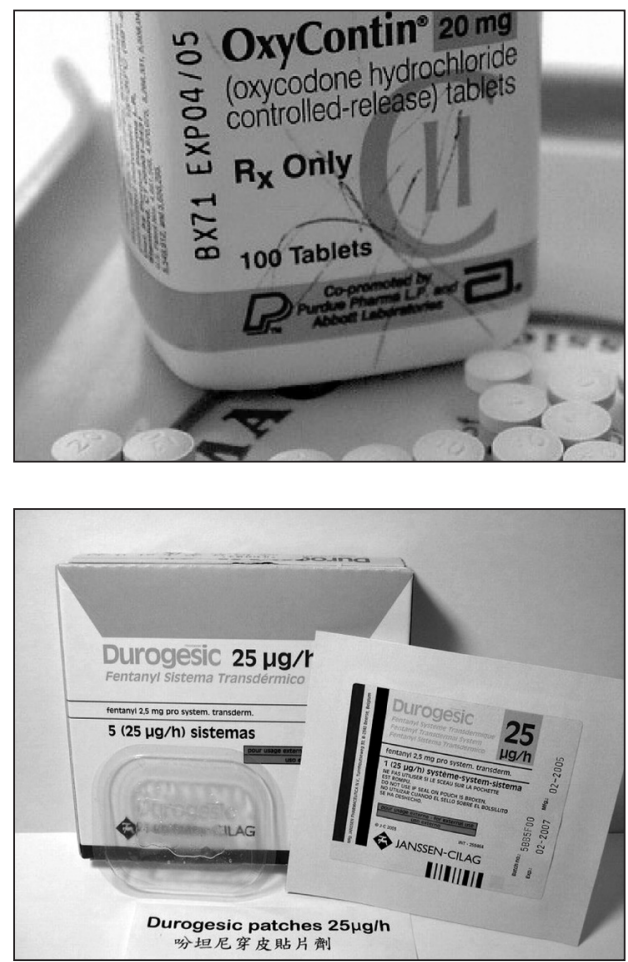

Figura 2. Presentaciones de nuevos opiáceos.

palpitaciones, arritmias cardíacas, hipertensión arterial, midriasis y sequedad de las mucosas. No hay un antídoto específico para esta intoxicación. El tratamiento consiste en proporcionar las medidas de soporte adecuadas, evitando que el paciente se autolesione y protegiéndole de los estímulos externos que pudieran agravar la sobreestimulación ya presente.

A la vista del potencial de abuso, creemos que se requieren estudios entre la población adolescente de nuestro país, que proporcionen información sobre el abuso del MTF. Además, se ha indicado la probabilidad de que exista el consumo/comercio de MTF por parte de padres o cuidadores de niños con este tratamiento, aspectos también a considerar entre los prescriptores de MTF.

\section{Modafinilo}

El modafinilo es un fármaco neuroestimulante sintético que se utiliza para la narcolepsia con o sin cataplejia y que me-
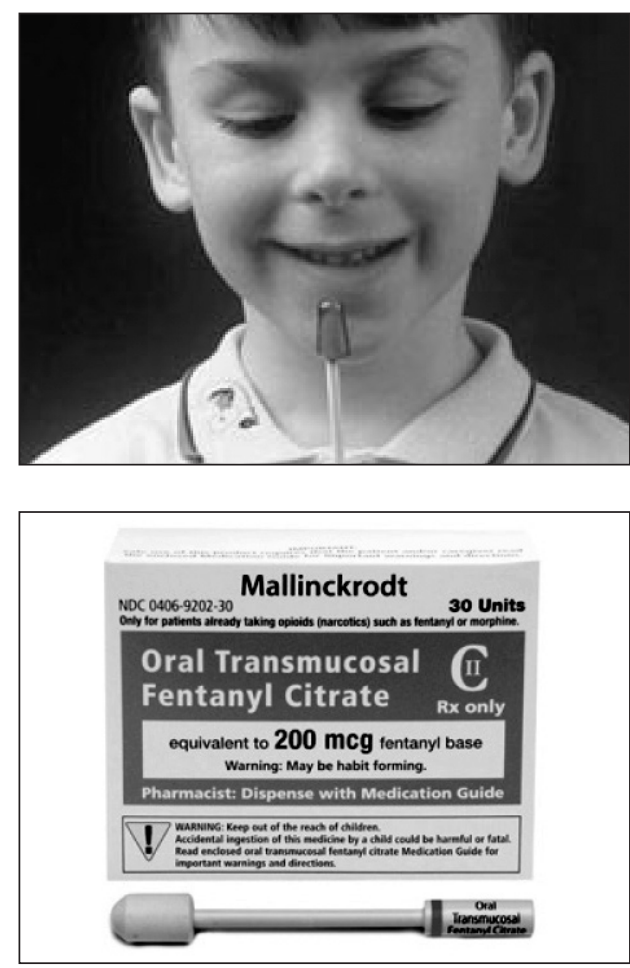

jora el nivel de vigilia y de alerta diurna, sin afectar al sueño durante la noche. No modifica la cataplexia ni los síntomas REM anómalos ${ }^{46}$. Fue autorizado en España en 1997 y su única indicación autorizada es la somnolencia excesiva asociada a la narcolepsia, existiendo una única especialidad farmacéutica, el Modiodol®. En otros países europeos y en EEUU, se pueden encontrar otras 10 especialidades farmaceúticas como Provigil@, Mentix ${ }^{\circledR}$, Vigil ${ }^{\circledR}$, Zalux ${ }^{\circledR}$, Alertec $\AA$ y Resotyl $\AA$.

Su mecanismo de acción es complejo y no está del todo esclarecido, asociándose la estimulación $\alpha$-adrenérgica y la interacción 5-HT/GABA. Parece que también pueda contribuir a una inhibición selectiva y de baja potencia de la recaptación de dopamina y a la estimulación de la actividad neuronal hipotalámica que libera orexina ${ }^{47}$. Respecto a sus propiedades farmacocinéticas, la absorción tras su administración oral es buena, alcanzándose la concentración plasmática máxima a las dos o tres 
horas de la ingestión. Se metaboliza en el hígado, principalmente a través del citocromo (CYP) 3A4, y su metabolito principal, el ácido modafinilo (40-50\% de la dosis) no tiene actividad farmacológica. La excreción de modafinilo y sus metabolitos es fundamentalmente renal, con una pequeña proporción que se elimina inmodificada $(<10 \%)$. Su semivida de eliminación es larga, en torno a las 12 horas $^{48}$.

Las reacciones adversas que se han descrito con mayor frecuencia son taquicardia, palpitaciones, somnolencia, confusión y dolor torácico y abdominal, entre otras. Con menor frecuencia, sin que por ello sean menos importantes, se han descrito espasmos musculares, descoordinación, síndrome de Stevens Johnson (sobre todo en población infantil), necrólisis epidérmica tóxica, amnesia, agresividad e ideación suicida ${ }^{49,50}$. También se han observado alteraciones en el ECG en forma de extrasístoles ventriculares ${ }^{51}$.

En niños con TDAH los ensayos clínicos realizados con modafinilo han mostrado la misma eficacia que con el metilfenidato, pero no se ha procedido a su autorización por parte de la FDA por la toxicidad dermatológica observada en ellos ${ }^{52}$. La realización de trabajos sobre el uso de modafinilo entre trabajadores sanitarios y personal militar, con el fin de valorar su efecto en mitigar la somnolencia y la fatiga asociados al trabajo en turnos nocturnos, encontraron que mejoraba sus capacidades manuales y cognitivas ${ }^{53,54}$, por lo que en 2004 la FDA amplió la indicación del modafinilo al trastorno del sueño en trabajadores por turnos y para la fatiga asociada a la apnea del sue$\tilde{\text { ño }}{ }^{55}$.

El potencial de abuso de este fármaco parece ser bajo, debido al retraso en alcanzarse su concentración plasmática máxima y producir así el efecto deseado ${ }^{42}$. Además, es poco hidrosoluble, por lo que su utilización por vía intravenosa no es posible. Su inestabilidad a temperatura elevada no permite su uso fumado. Para la compra de este medicamento se precisa de receta médica, aunque se puede obtener a través de internet, siendo sobre todo demandado por estudiantes universitarios. En julio de
2010, la Agencia Española del Medicamento y Productos Sanitarios (AEMPS) publicó una nota informativa acerca de la evaluación del riesgo/beneficio del uso del modafinilo, en la que también se hacía mención a la aparición en nuestro país de casos de abuso con fines recreativos ${ }^{50}$.

En la literatura existe poca información sobre la toxicidad producida por el abuso de modafinilo. Spiller y col estudiaron las llamadas realizadas a 15 centros de información toxicológica norteamericanos relacionadas con la ingesta de modafinilo como único tóxico entre 2000 y 2007. De los 137 casos que recogieron, el 31\% eran menores de seis años. El uso intencional fue la causa del 5\% de las consultas. El 66\% de los pacientes se derivaron al hospital, siendo dados de alta el 38\% directamente desde el Servicio de Urgencias, precisando ingreso en planta un $17 \%$ y en psiquiatría el $11 \%{ }^{56}$. En la serie de Carstairs y col, se analizaron en 11 años (1998-2008) las llamadas atendidas en el California Poison Control System. Los casos con ingesta única de modafinilo fueron 87 , un $20 \%$ en menores de seis años y el $6,9 \%$ por consumo recreacional. El $38 \%$ de los pacientes fueron derivados al servicio de Urgencias e ingresaron en la UCI el $4,6 \%{ }^{57}$.

En ambos estudios la mayoría de los efectos tóxicos fueron considerados leves (37\% y $62 \%$ respectivamente) o moderados (16\% y $12 \%)$. El principal síntoma encontrado fue la taquicardia (26-28\%) sin otras alteraciones del ECG, seguido de las síntomas de tipo neurológico (38\%-70\%) fundamentalmente agitación, ansiedad y cefalea, y la hipertensión arterial (4\%-7\%). No se describieron en estas dos publicaciones efectos tóxicos significativos con dosis inferiores a $400 \mathrm{mg} /$ ingesta, ni hubo fallecimientos. La dosis máxima ingerida fue de $6 \mathrm{~g}$ en un varón de 19 años que presentó agitación, delirio y desorientación. En la serie de Spiller y col se registró un caso de convulsión, y los síntomas y signos asociados al ingreso fueron fundamentalmente agitación, dolor torácico, distonías y aumento de $\mathrm{CPK}^{56}$. En los casos pediátricos, las dosis ingeridas fueron menores y en general los síntomas fueron más leves. No obstante, hay que re- 
saltar que ambos estudios fueron de tipo retrospectivo, sólo basados en llamadas (sin confirmación analítica) y referidos a casos de ingesta única (en la serie de Carstairs y col, las casos de ingesta combinada con otros tóxicos fueron 108, un 124\% más que los registros analizados) $)^{57}$. También se encuentran en la literatura dos abstracts de congresos, con pequeñas series de casos en población adulta ${ }^{58}$ y pediátrica ${ }^{59}$, con idénticos perfiles clínico-toxicológicos a las series comentadas, y un caso clínico de reacción adversa en una asociación de modafinilo y clozapina ${ }^{60}$. Recientemente, se ha descrito la ingesta de modafinilo en atletas de élite por el laboratorio antidopaje de la Federación Médico Deportiva Italiana ${ }^{61}$.

Debido a que la sintomatología que presenta la sobredosis de modafinilo con respecto a otro tipo de drogas de abuso es muy similar, su diagnóstico en el Servicio de Urgencias es complejo, además de no poderse detectar en muestras de orina o de plasma con las técnicas inmunoenzimáticas utilizadas habitualmente, por lo que la elaboración de una historia clínica bien dirigida será muy útil para la realización de un diagnóstico correcto.

\section{Dextrometorfano}

El dextrometorfano es el dextroisómero del opiáceo levorfanol y presenta actividad antitusígena. Ejerce su efecto terapéutico a través de su unión a receptores opioides kappa, mientras que no se une a receptores de tipo mu o delta, responsables de las actividades analgésicas y depresoras de los opiáceos. A dosis altas, su principal metabolito activo, el dextrorfano, puede actuar como antagonista competitivo del receptor NMDA (N-metil-D-aspartato), al igual que lo hacen la fenciclidina y la ketamina, farmacos conocidos por su uso como drogas estimulantes y disociativas ${ }^{33}$. Además, el dextrometorfano se une a receptores serotoninérgicos ${ }^{62}$.

Respecto a sus características farmacocinéticas, se absorbe bien por vía oral y alcanza su concentración máxima en 2-3 horas, aunque su actividad antitusígena se aprecia a los 15-30 minutos de su administración. Es metabolizado en el hígado, vía oxidativa, a través de la isoenzima CYP2D6 del citocromo P450, formándose derivados desmetilados como el dextrorfano. La semivida de eliminación del dextrometorfano es de 1,4 a 3,9 horas y del dextrorfano de 3,4 a 5,6 horas. Sin embargo, la semivida de eliminación puede llegar hasta 45 horas en los metabolizadores lentos ${ }^{63}$.

El dextrometorfano se encuentra comercializado en España desde 1958 y actualmente existen unas 50 especialidades farmacéuticas que lo contienen en forma de hidrobromuro. En algunas se encuentra como único principio activo y en otras, generalmente preparados antigripales, se asocia a vasoconstrictores como la pseudoefedrina, a antihistamínicos, expectorantes, analgésicos e incluso corticoides. La mayoría son especialidades farmacéuticas publicitarias con numerosas presentaciones (jarabe o solución oral, cápsulas, comprimidos, sobres) que se pueden adquirir sin receta médica y, en torno al 10\% de ellas contienen etanol como excipien$\mathrm{te}^{64}$. Son, por tanto, especialidades farmacéuticas baratas y fáciles de conseguir en cualquier oficina de farmacia.

Desde un punto de vista clínico, el dextrometorfano a dosis terapéuticas es bien tolerado, sin embargo, cuando se produce abuso se desarrollan efectos psicotrópicos debido a la acumulación de su metabolito el dextrorfan, y su acción sobre el receptor $\mathrm{NMDA}^{64}$. La sintomatología es variable y dosis-dependiente, pasando por distintos estados que comienzan por un ligero efecto estimulante (a dosis superiores a $2 \mathrm{mg} / \mathrm{kg}$ ), seguido de ilusiones y alucinaciones, hasta llegar a un estado disociativo (a dosis superiores a $7,5 \mathrm{mg} / \mathrm{kg}$ ) que puede llegar a ser completo, similar al que se experimenta con la ketamina. Estos efectos se acompañan de sensación de euforia, inquietud, pérdida de concentración y ataxia. Los efectos sobre el comportamiento comienzan a los 30-60 minutos de la ingestión y pueden durar hasta 6 horas. Dependen en gran medida de la dosis que el sujeto ingiera, pero también de su fenotipo metabólico, dado que los metabolizadores rápidos producen una mayor cantidad del metabolito activo responsable del efecto psicótropo. 
La ingestión aguda de dextrometorfano puede producir también efectos adversos como midriasis y nistagmus (típicamente rotatorio), vómitos con los preparados líquidos y en caso de ingestión masiva puede producirse depresión respiratoria, taquicardia e hipertensión arterial, habiéndose descrito casos mortales ${ }^{64,65}$. Estos y otros síntomas (hipertermia, retención urinaria, sequedad de piel y mucosas, midriasis, alteraciones de la motilidad gastrointestinal o delirio) pueden deberse a la ingestión de especialidades farmacéuticas que contienen no sólo dextrometorfano sino aminas simpaticomiméticas (pseudoefedrina), antihistamínicos anticolinérgicos (clorfeniramina) o etanol como excipiente ${ }^{57}$. Por sus propiedades pro-serotoninérgicas, el abuso de dextrometorfan se ha asociado ocasionalmente a la aparición de síndrome serotoninérgico, caracterizado clínicamente por alteración del estado mental, rigidez, hipertermia y convulsiones. El abuso crónico de dextrometorfano puede ocasionar el desarrollo de tolerancia, fenómenos de craving (deseo intenso de satisfacer una conducta adictiva) y dependencia de forma aislada, así como síndrome de abstinencia tras su retirada (vómitos, sudores nocturnos, mialgias, diarrea, escalofríos e inquietud $)^{65}$.

El abuso de dextrometorfano se conoce desde su comercialización en Estados Uni$\operatorname{dos}^{66}$. Sin embargo, en la última década se ha observado un aumento espectacular en su consumo, principalmente en adolescentes y adultos jóvenes. Se suele identificar entre sus consumidores por sus siglas DXM o por diversos nombres: Triple $\mathrm{C}$ (Coricidin Cough and Cold), dex, skittles (algunos comprimidos tienen un aspecto similar a dichos caramelos) y robo (por la especialidad Robitussin).

En el año 2006, la National Survey on Drug Use and Health (NSDUH) preguntó a personas de edad superior a los 12 años sobre su consumo de preparados over-thecounter (OTC) antitusígenos o antigripa$\operatorname{les}^{67}$. Los resultados mostraron que entorno a 3,1 millones de personas de 12 a 25 años (5,3\%) habían tomado alguna vez un preparado para la tos y el resfriado, como droga para encontrarse mejor, es decir de forma no terapéutica, y cerca de 1 millón $(1,7 \%)$ lo habían hecho durante el año anterior.

Según el informe de la DAWN, en 2004 un total de 16.858 visitas a los servicios de Urgencias se relacionaron con problemas ocasionados por el dextrometorfano, lo que supuso el $1 \%$ de todas las visitas relativas a fármacos ${ }^{68}$. La tasa anual de visitas a los servicios de urgencias por un uso no médico de dextrometorfano en el grupo de edad de 12 a 20 años fue de 8 visitas/100.000 sujetos frente a 2,5 visitas/ 100.000 sujetos en otros grupos de edad. Sin embargo, la ingesta accidental de dextrometorfano fue el principal problema en el grupo de edad de 0 a 11 años.

También este incremento en el consumo de dextrometrofano con fines no médicos puede constatarse en los informes del Sistema de Vigilancia de Exposición a Tóxicos (Toxic Exposure Surveillance System -TESS-) de la American Association of Poison Control Centres. Durante el periodo de 1999 a 2004 se atendieron en los US Poison Control Centres 15.543 casos de abuso de dextrometorfano como sustancia única. Un análisis pormenorizado de los 1.382 casos recibidos en California identificó un aumento de 10 veces en el número de casos recibidos desde 1999 (0,23 casos/1.000 llamadas) hasta 2004 (2,15 casos/1.000 llamadas). De los casos recibidos el $74,5 \%$ se produjo en el grupo de edad de 9 a 17 años, en el que el incremento de frecuencia en el periodo estudiado fue de 15 veces. La mayor parte de los casos mostraban síntomas leves (46\%) o moderados $(41,8 \%)$ y solo siete casos $(0,5 \%)$ fueron graves. El $20 \%$ de los sujetos mostraban consumo asociado de otras sustancias, siendo las más frecuentes el alcohol, la marihuana y los opiáceos ${ }^{69}$.

\section{Propofol}

La muerte de Michael Jackson sorprendió a muchos el 25 de junio de 2009 . $\mathrm{Al}$ parecer fue encontrado en su domicilio en parada cardiorrespiratoria, intentándole reanimar, primero su médico personal y luego una unidad de paramédicos, que 
finalmente lo traslado al Ronald Reagan UCLA Medical Center de los Ángeles donde fallece $^{70}$. Pero no menos sorprendente, ha sido para muchos sanitarios la presunta relación entre su fallecimiento y el abuso del anestésico propofol ${ }^{71}$. El empleo de propofol como droga de abuso es raro, aunque una revisión de los datos hasta ahora conocidos de su empleo con tal fin, no dejan de inducir a una profunda reflexión.

La mayoría de las actuales drogas de abuso (opioides, cocaína, anfetamina y derivados, LSD, ketamina, fenciclidina, gamma-hidroxibutirato, etc) han sido primero conocidas por su empleo en medicina. Tras su empleo con esta finalidad, han pasado a utilizarse como drogas de abuso o su uso ha producido farmacodependencia ${ }^{72,73}$.

El propofol es un fármaco de administración intravenosa, muy utilizado en anestesia, medicina intensiva y endoscopia entre otras actividades médicas, para inducir sedación e hipnosis ${ }^{74}$. Sus principales características son un rápido inicio del efecto, una corta duración de sus acciones y escasas reacciones adversas ${ }^{75}$. Tradicionalmente no ha sido considerado una droga de abuso, sin embargo con dosis subanestésicas se describió un hipotético empleo potencial con este fin ${ }^{76}$. A pesar de su amplia utilización en todo el mundo, no se han descrito casos de farmacodependencia en pacientes en los que se usa propofol para su sedación o anestesia. Desde su comercialización en el año 1980 hasta el 2007, sólo se habían notificado en la literatura médica 9 casos de abuso o dependencia, el primero en 1992, correspondiendo cuatro de ellos a fallecimientos ${ }^{77}$. Todas las muertes fueron por insuficiencia respiratoria tras una apnea. Siete de los nueve casos de abuso de propofol ocurrieron en médicos generalistas o anestesistas ${ }^{78,79}$, personal de enfermería $^{80,81}$, auxiliar de quirófano ${ }^{82,83} \mathrm{u}$ otros sanitarios ${ }^{84}$.

En el año 2007 se publicó una encuesta sobre el abuso de propofol, realizada en 126 hospitales con programa de formación de médicos residentes en anestesiología en EE.UU. Se notificó que un 18\% (25 casos) de los servicios de anestesia había tenido uno o más incidentes de abuso de propofol en los 10 años previos. De los 25 sanitarios con problemas de abuso del propofol, 7 habían muerto (28\%) como consecuencia de su empleo (6 de ellos médicos residentes). En todos los casos de muerte, el abuso de propofol fue descubierto a consecuencia del fallecimiento. El 71\% de los hospitales estudiados no tenía un sistema de control de la utilización de propofol. En 2008 y 2009, los casos publicados en la literatura médica, implican también al personal sanitario ${ }^{85,86}$. Aparentemente, con la excepción de Michael Jackson, sólo había hasta la fecha un caso de personal no médico con problemas de abuso de propofol ${ }^{75}$. Por otro lado, el único caso descrito en toda la literatura médica de homicidio utilizando propofol por parte de un enfermero, se cometió en EE.UU en $2008^{87}$.

Así pues, debemos preguntarnos si está emergiendo un fenómeno de abuso de propofol, que hasta ahora no habíamos tenido en cuenta y del que sólo conocíamos la punta del iceberg ${ }^{88}$. La próxima llegada de profármacos del propofol (fospropofol), hidrosolubles y por tanto con una mayor biodisponibilidad $^{89}$, nos hace además plantearnos la posibilidad de que irrumpan en el espectro del pharming un nuevo tipo de sustancias a considerar como potenciales $\operatorname{drogas}^{90}$.

\section{Nuevos opiáceos}

Los opiáceos son los fármacos más potentes que se comercializan para el alivio del dolor agudo o crónico ${ }^{91}$. Se ha producido una enorme difusión del uso de opiáceos sintéticos tanto para el tratamiento del dolor oncológico como para el del dolor no oncológico, agudo o crónico. El uso de opiáceos sintéticos (tramadol, fentanilo, oxicodona) en formatos cómodos de uso (parches, comprimidos, dispersables, chupa-chups) y su difusión en el tratamiento del dolor, está produciendo un aumento de los problemas relacionados con su uso, tanto en sus efectos adversos propiamente dichos como en la generación de abuso y dependencia ${ }^{92}$. Además, se ha constatado en Estados Unidos un aumento en el desvío de opiáceos de prescripción médica a uso 
recreativo, así como la comercialización a través de internet de copias ilegales de estos fármacos ${ }^{93}$. Los patrones de consumo cambian según el país, constituyendo el principal problema emergente en EEUU, donde el fentanilo es la segunda droga más frecuentemente asociada con mortalidad e intoxicaciones graves. En las repúblicas bálticas, hay indicios de un problema creciente causado por el 3-metilfentanilo y su uso ilícito. Recientemente se ha informado en Estonia de 46 intoxicaciones mortales en 2005 y 71 en 2006. En 2007, dos tercios de los usuarios de drogas vía parenteral encuestados en Estonia, confirmaron el uso de fentanilo como su droga principal ${ }^{94}$.

El proyecto Euro-TREND ha advertido del consumo de heroína y medicamentos opiáceos en colectivos nuevos, entre los que se encuentran personas socialmente integradas y público que acude a festivales 'tecno'. En estos grupos puede haber consumidores experimentales y personas capaces de controlar su consumo de dro$\mathrm{ga}^{95}$.

En nuestro medio, el principal problema emergente es el abuso y dependencia a opiáceos sintéticos instaurada de forma iatrogénica en la población general. Existe un aumento considerable de la prescripción de opiáceos sintéticos para el tratamiento de dolor crónico no oncológico (artrosis, lumbalgias, hernias discales, fibromialgia, etc) en atención primaria, traumatología, unidades del dolor y otras especialidades. Esto comporta un aumento de asistencias en los servicios de urgencias por problemas relacionados con el tratamiento, sobre todo en pacientes pluripatológicos, polimedicados y geriátricos ${ }^{96}$. También se está produciendo un aumento de la demanda de desintoxicación por dependencia a opiáceos sintéticos, lo que plantea problemas por la comorbilidad orgánica y psiquiátrica, interacciones farmacológicas y dificultad para la desintoxicación.

El fentanilo, junto al formato en ampollas para uso en anestesia, se presenta en forma de parches de liberación prolongada (72h) con dosis de 25 a $100 \mu \mathrm{g} / \mathrm{h}$ y chupachups de liberación bucal con dosis de 200 a $1600 \mu \mathrm{g}$, de absorción inmediata y mayor potencial de abuso. Se ha descrito el abuso de fentanilo con intención recreativa en todos sus formatos, pero predominan los productos transdérmicos y dispersables, que se ingieren, mastican, disuelven en agua, fuman, inyectan e incluso se insertan por vía rectal. Además, tras finalizar el uso de los parches transdérmicos aún queda una cantidad de principio activo suficientemente alta, siendo utilizados estos parches de fentanilo "de segunda mano" como sustancias de abuso ${ }^{97}$.

En EEUU ha habido numerosos casos de muerte por intoxicación por fentanilo, habiéndose utilizado mezclado con heroína ${ }^{98}$. Como nombres callejeros destacan: "drop dead", "flat Line", "lethal injection", "China White", "heroína sintética", "Apache", "Osa Mayor", "Tango \& Cash", "TNT", etc. Si el poder analgésico del fentanilo es 470 superior a la morfina, el alfa-metilfentanilo es 600 veces más potente, el 3-metil fentanilo 6.684 veces y el carfentanilo 10.000 veces más potente que la morfina.

Un número significativo de muertes se han registrado en la UE y los EEUU tras la ingesta de modalidades de fentanilo sintetizados ilegalmente, denominados fentanilo no farmacéuticos. Algunas muertes han sido causadas por el consumo de heroína mezclada con fentanilo o con alguno de sus análogos más potentes, como el alfa-metilfentanilo y el 3 metilfentanilo ${ }^{99}$.

La oxicodona es otro opioide semisintético derivado de la tebaína (alcaloide del opio), que presenta sinergia con la morfina (oxicodona $10 \mathrm{mg}$ equivalen a morfina 20 $\mathrm{mg}$ ). Es un agonista puro mu y kappa con acción analgésica, ansiolítica y sedante. Se encuentra comercializada en comprimidos de liberación prolongada (5-80 mg) o inmediata (5-20 mg). Su dosificación respectiva es cada 12 horas o cada 4-6 horas. Presenta menor metabolismo de primer paso que la morfina, con metabolización hepática por citocromo P450 2D6 y eliminación renal. No existen metabolitos activos.

Presenta una menor prevalencia de efectos secundarios en comparación con la morfina (menos náuseas, trastornos cognitivos y prurito), pero puede producir intoxicación con depresión respiratoria. Aunque 
en nuestro medio es un fármaco de difusión limitada, en otros países como EEUU donde es conocida como "Oxy", "Oxycotton", "OC", "hillbilly heroin" o "heroína de los pobres", el abuso de oxicodona constituye un problema de salud pública, con una mortalidad importante ${ }^{100}$. También se utiliza por vía nasal y habitualmente junto al consumo de otras sustancias, fundamentalmente benzodiacepinas y alcohol.

La buprenorfina es un agonista parcial mu y antagonista kappa, utilizada para el tratamiento de la adicción a opiáceos y como analgésico. Puede producir a dosis altas depresión del SNC y respiratoria. Presenta un metabolismo primer paso, con metabolización hepática por el citocromo CYP3A4 y metabolito activo (norbuprenorfina) que prolonga los efectos.

Se usa por vía sublingual, con una vida media de 4-5 h y en parches de liberación retardada. La asociación buprenorfinanaloxona se utiliza para evitar el abuso por vía parenteral de la buprenorfina y es preferible este uso combinado que la buprenorfina sola, excepto en el embarazo. Se han descrito recientemente en Europa casos de intoxicaciones por abuso de buprenorfina ${ }^{101,102}$. En Finlandia constituye el primer opiáceo en demanda de tratamiento de desintoxicación.

Existen otros fármacos opiáceos como el tramadol, hidromorfona, petidina, codeína y metadona utilizados tanto para el tratamiento del dolor como en la adicción a opiáceos, habiéndose descrito abuso de los mismos por pacientes con dependencia a opiáceos ${ }^{10}$.

En el abordaje terapéutico de la intoxicación aguda por fármacos opiáceos sintéticos se debe actuar de forma general como en cualquier sobredosis por opiáceos con las medidas básicas de reanimación cardiopulmonar y uso de naloxona, pero se deben tener en cuenta unas premisas: valorar la posibilidad de policonsumo de sustancias que pueden alterar el cuadro clínico y condicionar el tratamiento, la duración de los efectos tóxicos en pacientes que han consumido preparados de liberación prolongada, la posible intoxicación concomitante por paracetamol cuando está incluido en el preparado farmacéutico analgésico ${ }^{35}$, y la aparición de complicaciones secundarias a la intoxicación aguda (neumonías por broncoaspiración, edema agudo de pulmón no cardiogénico, rabdomiolisis aguda y necrosis tubular aguda, descompensación de otras patologías, etc) ${ }^{103}$.

A las sustancias descritas se podrían añadir varios grupos de fármacos, aunque por la información disponible y por la frecuencia observada en otros continentes, fundamentalmente USA, parece que su uso todavía es testimonial. Así, hay indicios del uso de pregabalina con finalidad recreativa, con casos observados en los países escandinavos, incluso en forma endovenosa, uso oral de benzidamida, de gabapentina, sildenafilo con finalidad lúdica entre sujetos jóvenes sin disfunción eréctil, y de las comentadas "z-drugs", entre deportistas de élite ${ }^{104,105}$. En cualquier caso habrá que estar atentos a las modas y tendencias detectadas en las encuestas y, sobre todo los clínicos a los casos que los clínicos vayan detectando en su trabajo, para lo que se requiere un alto nivel de sospecha y pensar en esta forma de consumo que representa el pharming.

\section{BIBLIOGRAFÍA}

1. LEVINE DA. "Pharming": the abuse of prescription and over-the-counter drugs in teens. Curr Opin Pediatr 2007; 19: 270-274.

2. Prosser JM, Nelson LS. Trends in adolescent drug abuse. Emerg Med 2008; 40: 8.

3. WAX PM. Just a click awai: recreational drug web sites on the internet. Pediatrics 2002; 109: 96.

4. Naciones Unidas: Junta Internacional de Fiscalización de Estupefacientes. Informe de la Junta Internacional de Fiscalización de Estupefacientes correspondiente a 2010. Disponible en URL: http://www.incb.org/pdf/annualreport/2010/es/Spanish_pdf.pdf. Acceso el $1 / 08 / 2012$.

5. Burillo-Putze G, Climent Díaz B, Medina Vega L, Toledo Ravelo A. Las smart-drugs: un nuevo reto clínico en urgencias y emergencias. Emergencias 2010; 22: 477-478.

6. Burillo-Putze G, Climent B, Echarte JL, Munné P, Miró O, Puiguriguer J, Dargan P. Drogas emergentes (I): las "smart drugs". Anales Sist Sanit Navar 2011; 34: 263-274. 
7. Maxwell JC, Coleman JJ, Feng SY, Goto CS, TiRaDo CF. Cheese: An old drug in a new wrapper. Drug Alcohol Depend 2012; 126: 161-167.

8. Center for Behavioral Health Statistics and Quality, Substance abuse and Menth Helath Services Administration. Highlights of the 2009 Drug Abuse Warning Network (DAWN) Findings on Drug-Related Emergency Department Visits. Disponible en URL: http:// www.oas.samhsa.gov/2k10/DAWN034/EDHighlightsHTML.pdf. Acceso 2/08/2012.

9. Azkunaga B, Mintegi S, del Arco L, Bizkarra I. Cambios epidemiológicos en las intoxicaciones atendidas en los servicios de urgencias pediatricos españoles observados entre 2001 y 2010: incremento de las intoxicaciones por etanol. Emergencias 2012; 24: 376-379.

10. Clemente Rodríguez C, Echarte Pazos JL, Aguirre Tejedo A, Puente Palacios I, Iglesias Lepine ML, Supervía Caparrós A. Diferencias en las intoxicaciones de los españoles y los extranjeros atendidas en urgencias. Emergencias 2011; 23: 271-275.

11. Delegación del Gobierno para el Plan Nacional sobre Drogas. Encuesta domiciliaria sobre alcohol y drogas en España (EDADES), 1995-2007. http://www.pnsd.msc.es/Categoria2/observa/pdf/Domiciliaria2007.pdf

12. Delegación del Gobierno para el Plan Nacional sobre Drogas. Encuesta domiciliaria sobre alcohol y drogas en España (EDADES), 2009-2010. http://www.mspsi.es/gabinetePrensa/notaPrensa/pdf/presentacionEdades200910.ppt

13. OMA butlletí núm 1. Barcelona: Col-legi Oficial de Farmacèutics de Barcelona; 2006.

14. Brugada J. Papel de los urgenciólogos en la detección de pacientes con riesgo de muerte súbita. Emergencias 2011; 23: 5-7.

15. LubelcheK RJ, Kroc KA, Levine DL, Beavis KG, ROBERTS RR. Routine, rapid HIV testing of medicine service admissions in the emergency department. Ann Emerg Med. 2011; 58: 65-70.

16. Burillo-Putze G, Borreguero León JM, Vallbona Afonso E, De Vera González AM, Fernández RoDríguez JF, García Dopico JA, et al. A Consumo de cocaína y su relación con patología cardiaca y traumática atendida en un servicio de urgencias. Emergencias 2008; 20: 380-384.

17. Clemente Rodríguez C, Puente Palacios I, López Casanova MJ, laso de la Vega i Artal S, Aranda Cárdenas D, Puiggalí Ballart M. Efecto de la educación sanitaria en la demanda de anticoncepción de urgencias. Emergencias 2011; 99-103.

18. Carbajosa-Dalmau J, Llorens P, Diéguez-Zaragoza S, Carratalá-Perales JM, Díaz-Dámaso J, MartíNEZ-BELoqui E, et al. Influencia del sexo del pa- ciente en el manejo del síndrome coronario agudo con elevación del ST en los servicios de urgencias. Emergencias 2011; 23: 87-92.

19. Hernández Sánchez MJ, Segura Clavell J, BuriLlo-Putze G. Papel clave de los servicios de emergencias en la tragedia de la inmigración ilegal por vía marítima. Emergencias 2008; 20: $377-379$

20. Castro Delgado R, Arcos González P, Cuartas AlVAREz T. Desastres e intoxicación por monóxido de carbono. Emergencias 2011; 335-336.

21. Pinillos MA, Ostolaza PB. Urgencias, año cero. An Sist Sanit Navar 2011; 34: 343-348.

22. La importancia de adecuar la investigación sobre el uso de los servicios sanitarios al objetivo perseguido. Emergencias 2011; 23: 266-270.

23. Espinosa G, Miró O, Nogué S, To-Figuera J, SánCHEz M, Coll-Vinent B. Intoxicación por éxtasis líquido: análisis de 22 casos. Med Clin (Barc) 2001; 117: 56-58

24. Anta Fernández M, Bravo González JM, Fernández Rozas S, Goffaux Gómez-Caro O, García-Castrillo RIESGo L. Escombrointoxicación por consumo de bonito. Emergencias 2001; 13: 132-135.

25. Miró O, Rovira E, Blanco AJ, Salmerón JM, GATELl JM, CERVERA C, et al. Descripción clínica y epidemiológica de los primeros casos de la gripe nueva A (H1N1) atendidos en España. Emergencias 2009; 21: 166-171.

26. Burillo Putze G, Munné Mas P, Dueñas laita A, Trujillo Martín MM, Jiménez Sosa A, Adrián MARTín MJ, et al. Intoxicaciones agudas: perfil epidemiológico y clínico, y análisis de las técnicas de descontaminación digestiva utilizadas en los servicios de urgencias españoles en el año 2006 -Estudio HISPATOX-. Emergencias 2008; 20: 15-26.

27. YIN S, WAHL M. Intentional Coricidin product exposures among Illinois adolescents. Am J Drug Alcohol Abuse 2011; 37: 509-514.

28. Sogut O, Kaya H, Gokdemir MT, Sezen Y. Acute myocardial infarction and coronary vasospasm associated with the ingestion of cayenne pepper pills in a 25 -year-old male. Int $\mathrm{J}$ Emerg Med 2012; 5: 5.

29. Yin S, WAHL M. Intentional Coricidin product exposures among Illinois adolescents. Am J Drug Alcohol Abuse 2011; 37: 509-514.

30. La saturación de los servicios de urgencias: una llamada a la unidad. Emergencias 2011; 23: 59-64.

31. Ovens H. Saturación de los servicios de urgencias. Una propuesta desde el Sistema para un problema del Sistema. Emergencias 2010; 22: 244-246. 
32. Cimonali N. Zopiclone: is it a pharmacologic agent for abuse? Can Fam Physician 2007; 53 : 2124-2129.

33. Abanades S, Peiró AM, Farré M. Club drugs: los viejos fármacos son las nuevas drogas de la fiesta. Med Clin (Barc) 2004; 123: 305-311.

34. Galicia M, Nogué S, To-Figueras J, Echarte JL, IgLESIAS ML, MiRó O. Intoxicaciones por éxtasis líquido atendidas en servicios de urgencias hospitalarios de la ciudad de Barcelona durante 2 años. Med Clin (Barc) 2008; 130: 254-258.

35. García Andrés MC, Arias Rodríguez A, Rodríguez Álvarez C, Morcillo Rehberger A, Aguirre-Jaime J. Análisis de series temporales aplicado a intoxicaciones atendidas en urgencias hospitalarias. Emergencias 2011; 23: 193-199.

36. Leonard BE, McCartan D, White J, King DJ. Methylphenidate: a review of its neuropharmacological, neuropsychological and adverse clinical effects. Hum Psychopharmacol 2004; 19: 151-80.

37. Nota informativa de la AEMPS. Ref: 2009/01: Metilfenidato: actualización de las condiciones de uso.

38. VolKow ND, Swanson JM. Variables That Affect the Clinical Use and Abuse of Methylphenidate in the Treatment of ADHD Am J Psychiatry 2003; 160: 1909-1918.

39. Diario El Pais. La chuleta que viene en pastillas. Disponible en URL: http://sociedad. elpais.com/sociedad/2010/06/12/actualidad/1276293602_850215.html

40. Teter CJ, McCabe SE, LaGrange K, Cranford JA, Boyd CJ. Illicit Use of Specific Prescription Stimulants Among College Students: Prevalence, Motives, and Routes of Administration. Pharmacotherapy 2006; 26: 1501-1510.

41. Poulin C. Medical and nonmedical stimulant use among adolescents: from sanctioned to unsanctioned use. CMAJ 2001; 165: 1039-1044.

42. Setlik J, Bond R, Mona H. Adolescent Prescription ADHD Medication Abuse Is Rising Along With Prescriptions for These Medications. Pediatrics 2009; 124; 875-880.

43. Morton WA, Stockton GG. Methylphenidate abuse and psychiatric side effects. Prim Care Companion J Clin Psychiatry 2000; 2: 159-164.

44. McCormick TC JR, McNeel TW. Acute psychosis and ritalin abuse. Tex State J Med 1963; 59: 99-100.

45. JAFFE SL. Intranasal abuse of prescribed methylphenidate by an alcohol and drug abusing adolescent with ADHD. J Am Acad Child Adolesc Psychiatry 1991; 30: 773-775.
46. KeAm S, WaLKer MC. Therapies for narcolepsy with or without cataplexy: evidence based review. Curr Opin Neurol 2007; 20: 699-703.

47. Flores J, Armijo JA, Mediavilla Á. Farmacología Humana: Barcelona: Edición Elsevier Masson; 2008. p. 564.

48. Ficha Técnica del modiodal ${ }^{\circledR}$, http://www. aemps.gob.es/cima/especialidad.do?metod $\mathrm{o}=$ verFichaWordPdf\&codigo=61540\&formato =pdf\&formulario=PROSPECTOS

49. Malcolm R, Book SW, Moak D, DeVane L, CzePOWICZ V. Clinical applications of modafinil in stimulant abusers: low abuse potential. Am J Addict 2002; 11: 247-249.

50. Agencia Española de Medicamentos y productos sanitarios. MODAFINILO: EVALUACIÓN DE SU BALANCE BENEFICIO/RIESGO. NOTA INFORMATIVA 22 de julio de 2010 , Ref: 2010/09. Disponible en URL: http://www. aemps.gob.es/informa/notasInformativas/ medicamentosUsoHumano/seguridad/2010/ docs/NI_2010-09_modafinilo.pdf

51. Oskooliar N. A case of premature ventricular contractions with modafinil. Am J Psychiatry 2005; 162: 1983-1984.

52. KumAR R. Approved and investigational uses of modafinil: an evidence-based review. Drugs 2008; 68: 1803-1839.

53. NELSon D. Prevention and Treatment of Sleep Deprivation Among Emergency Physicians. Pediatr Emerg Care 2007; 23: 498-503.

54. Czeisler CA, Walsh JK, Roth T, Hughes RJ, Wright KP, KInGSBURY L et al. Modafinil for Excessive Sleepiness Associated with Shift-Work Sleep Disorder. N Engl J Med 2005; 353: 476-486.

55. Westcotт KJ. Modafinil, Sleep Deprivation, and Cognitive Function in Military and Medical Settings. Mil Med 2005; 170: 333-335.

56. Spiller HA, Borys D, Griffith JR, Klein-Schwartz W, Aleguas A, Sollee D, et al. Toxicity from modafinil ingestion. Clin Toxicol 2009; 47: 153156.

57. Carstairs SD, Urquhart A, Hoffman J, Clark RF, CANTRell FL. A Retrospective review of supratherapeutic modafinil exposures. J Med Toxicol 2010; 6: 307-310.

58. LACKEY GD, Alsop JA, Albertson TE. A 24 months retrospective study of adult modafinil ingestions. Clin Toxicol 2007; 45: 641.

59. Lackey GD, Alsop JA, SAnds TR, Albertson TE. A two years retrospective study of pediatric modafinil ingestions. Clin Toxicol 2007; 45: 643.

60. Dequardo JR. Modafinil-associated clozapine toxicity. Am J Psychiatry 2002; 159: 1243-1244.

61. Strano Rossi S, Botrè F. Prevalence of illicit drug use among the Italian athlete popula- 
tion with special attention on drugs of abuse: A 10-year review. J Sports Sci 2011; 29: 471-476.

62. Romanelli F, Smith KM. Dextromethorphan abuse: clinical effects and management. J Am Pharm Assoc 2009; 49: 20-25.

63. BOT Plus web. Dextrometorfano. Consejo General de Colegios Oficiales de Farmacéuticos. Disponible en URL: https://botplusweb. portalfarma.com/

64. BOyer EW. Dextromethorphan abuse. Pediatr Emerg Care. 2004; 20: 858-863.

65. Logan BK, Goldfogel G, Hamilton R, Kuhlman J. Five deaths resulting from abuse of dextromethorphan sold over the internet. J Anal Toxicol 2009; 33: 99-103.

66. Chyka PA, Erdman AR, Manoguerra AS, Christianson G, Booze LL, Nelson LS, et al.; American Association of Poison Control Centers. Dextromethorphan poisoning: an evidencebased consensus guideline for out-of-hospital management. Clin Toxicol (Phila) 2007; 45: 662-677.

67. Substance Abuse and Mental Health Services Administration, Office of Applied Studies. The NSDUH Report: Misuse of Over-theCounter Cough and Cold Medications among Persons Aged 12 to 25. Disponible en URL: http://www.samhsa.gov/data/2k8/cough/ cough.htm

68. Ball, JK, Albright, V. Substance Abuse and Mental Health Services Administration, Office of Applied Studies. Emergency department visits involving dextromethorphan. The New DAWN report 2006; 32: 1. Disponible en URL: http://www.oas.samhsa.gov/DAWN/dextromethorphan.cfm

69. Bryner JK, Wang UK, Hui JW, Bedodo M, MacDougall C, Anderson IB. Dextromethorphan abuse in adolescence: an increasing trend: 1999-2004. Arch Pediatr Adolesc Med 2006; 160: 1217-1222.

70. Diario El Pais. Fallece el cantante Michael Jackson. Disponible en URL: http:// elpais.com/elpais/2009/06/25/actualidad/1245912536_850215.html

71. Diario El Pais. Jackson murió por una sobredosis de calmantes. Disponible en URL: http://elpais.com/elpais/2009/08/24/actualidad/1251096535_850215.html

72. Haroz R, Greenberg MI. New drugs of abuse in North America. Clin Lab Med 2006; 26: 147164.

73. Ries RK, Millar SC, Hellin DA, Sainz R (Eds). Principles of Addiction Medicine. 3rd ed. Chevy Chase, MD:; 2007 Principles of Addiction Medicine, Fourth Edition. American So- ciety of Addiction Medicine. Lippincott Williams \& Wilkins, 2009.

74. Belinchón De Diego E, Belinchón De Diego A, Martínez Alcaraz A, Nogués Tomás S. Experiencia en el empleo de anestesia regional para la reducción de las luxaciones de hombro en urgencias. Emergencias 2011; 23: 303-6.

75. Fritz GA, Niemczyk WE. Propofol dependency in a lay person. Anesthesiology 2002; 96: 505506.

76. Zacny JP, Lichtor JL, Zaragoza JG, CoAlson DW, Uitvlugt AM, Flemming DC, et al. Assessing the behavioral effects and abuse potential of propofol bolus injections in healthy volunteers.Drug Alcohol Depend 1993; 32: 45-57.

77. Roussin A, Montastruc JL, Lapeyre-Mestre M. Pharmacological and clinical evidences on the potential for abuse and dependence of propofol: a review of the literature. Fundam Clin Pharmacol 2007; 21: 459-466.

78. Follette JW, FARLEy WJ. Anesthesiologist addicted to propofol. Anesthesiology 1992; 77: 817-818

79. Soyka M, Schutz CG. Propofol dependency. Addiction 1997; 92: 1369-1370.

80. Iwersen-Bergmann S, Rösner P, Kühnau HC, Junge M, Schmold A. Death after excessive propofol abuse. Int J Legal Med 2001; 114: 248-251.

81. Roussin A, Mirepoix M, Lassabe G, Dumestre-Toulet V, Gardette V, Montastruc JL, et al. Death related to a recreational abuse of propofol at therapeutic dose range. Br J Anaesth 2006; 97: 268 .

82. Drummer OH. A fatality due to propofol poisoning. J Forensic Sci 1992; 37: 1186-1189.

83. Odell M. Propofol abuse. Anaesth Intensive Care 1999; 27: 539

84. Cirimele V, Kintz P, Doray S, Ludes B. Determination of chronic abuse of the anaesthetic agents midazolam and propofol as demonstrated by hair analysis. Int J Legal Med 2002; 116: 54-57.

85. Bonnet U, Harkener J, Scherbaum N. A case report of propofol dependence in a physician. J Psychoactive Drugs. 2008; 40: 215-217.

86. Riezzo I, Centini F, Neri M, Rossi G, Spanoudaki E, Turillazzi E, Fineschi V. Brugada-like EKG pattern and myocardial effects in a chronic propofol abuser. Clin Toxicol (Phila) 2009; 47: 358-363.

87. Kirby RR, ColaW JM, Douglas MM. Death from propofol: accident, suicide, or murder? Anesth Analg 2009; 108: 1182-1184.

88. Wilson C, Canning P, Caravati EM. The abuse potential of propofol. Clin Toxicol (Phila) 2010; 48: 165-170. 
89. Fechner J, Schwilden H, Schüttler J. Pharmacokinetics and pharmacodynamics of GPI 15715 or fospropofol (Aquavan injection) - a water-soluble propofol prodrug. Handb Exp Pharmacol 2008; 182: 253-266.

90. Drug Enforcement Administration, Department of Justice. Schedule of controlled substances; placement of fospropofol into schedule IV. Final rule. Fed Regist 2009; 74 : 51234-51236.

91. Casal Codesido JR, Vázquez lima MJ. Abordaje del dolor musculoesquelético en urgencias. Emergencias 2012; 24: 59-65.

92. European Monitoring Centre for Drugs and Drug Addiction. Informe anual 2009: el problema de la drogodependencia en Europa, Oficina de Publicaciones de la Unión Europea, Luxemburgo. Disponible en URL: http:// www.emcdda.europa.eu/attachements.cfm/ att_93236_ES_EMCDDA_AR2009_ES.pdf

93. KATZ N. Internet-based survey of nonmedical prescription opioid in the United States. Clin J Pain 2008; 24: 28-35.

94. European Monitoring Centre for Drugs and Drug Addiction. Informe anual 2010: el problema de la drogodependencia en Europa, Oficina de Publicaciones de la Unión Europea, Luxemburgo. Disponible en URL: http:// www.emcdda.europa.eu/attachements.cfm/ att_120104_ES_EMCDDA_AR2010_ES.pdf

95. SHEWAN D, DALGaRno P. Evidence for controlled heroin use? Low levels of negative health and social outcomes among non-treatment heroin users in Glasgow (Scotland). $\mathrm{Br} \mathrm{J}$ Health Psychol 2005; 10: 33-48.

96. Iniesta Navalón C, Urbieta Sanz E, Gascón Canovas JJ, Madrigal de Torres M, Piñera Salmerón P. Evaluación de la anamnesis farmacoterapéutica realizada en el servicio de urgencias al ingreso hospitalario. Emergencias 2011; 23: 365-371.
97. Nelson L, Schwaner R. Transdermal fentanyl: pharmacology and toxicology. J Med Toxicol 2009; 5: 230-241.

98. Centers for Disease Control and Prevention, USA. Nonpharmaceutical fentanyl-related deaths-multiple States, April 2005-March 2007. Morbidity and Mortality Weekly Report, 2008; 57(29); 793-6. Disponible en URL: http://www.cdc.gov.mmwr/preview/mmwrhtml/mm5729a1.htm

99.Bronstein AC, Spyker DA, Cantilena LR JR, GreEN JL, Rumack BH, DART RC. 2010 Annual Report of the American Association of Poison Control Centers' National Poison Data System (NPDS): 28th Annual Report. Clin Toxicol (Phila) 2011; 49: 910-941.

100. Aquina CT, Marques-Baptista A, Bridgeman P, Merlin MA. Oxycontin abuse and overdose. Postgrad Med 2009, 121:163-167.

101. Mendelson J, Flower K, Pletcher MJ, Galloway GP. Addiction to prescription opioids: characteristics of the emerging epidemic and treatment with buprenorphine. Exp Clin Psychopharmacol 2008; 16: 435-441.

102. SulLIVAN LE. Narrative review: buprenorphine for opioid-dependent patients in office practice. Ann Intern Med 2008; 148: 662-670.

103. Boyer EW. Management of Opioid Analgesic Overdose. NEJM 2012; 367: 146-155.

104. Psychonaut Web Mapping Research Group (2010). Psychonaut Web Mapping Project: Final report. Institute of Psychiatry, King's College London: London UK. Disponible en URL: http://www.psychonautproject.eu/documents/reports/Psychonaut_Project_Executive_Summary.pdf

105. Mondón S, Nogué S, Urbano D, Rovira E. Adiccion a la gabapentina. Med Clin (Barc) 2010; 134: 138-139. 